\section{La Estrategia de Gestión Integrada para la Prevención y el Control del Dengue en la Región de las Américas}

\author{
José Luis San Martín \\ y Olivia Brathwaite-Dick'
}

Palabras clave: dengue, estrategias regionales, estrategias nacionales, prevención primaria, Américas.

\footnotetext{
1 Programa Regional de Dengue, Unidad de Control de Enferme-dades Transmisibles, Organización Panamericana de la Salud/ Organización Mundial de la Salud, Panamá, República de Panamá. La correspondencia se debe dirigir a José L. San Martín, Programa Regional de Dengue, Unidad de Control de Enfermedades Transmisibles, Organización Panamericana de la Salud/Organización Mundial de la Salud, Ave. Gorgas, Edificio 261, Ministerio de Salud, Piso 2, Apartado 0816-02862, Panamá, República de Panamá. Correo electrónico: sanmarjl@pan.ops-oms.org
}

El dengue constituye un problema creciente de salud pública en la Región de las Américas y el mundo, y por el elevado número de personas afectadas está considerada la enfermedad vírica más importante transmitida por artrópodos (1). La reaparición y la gravedad del dengue están asociadas con los llamados macrofactores (ambientales, socioeconómicos, políticos y sociales) $(2,3)$ y microfactores (dependientes de las características biológicas del virus, el vector y la persona afectada).

Entre los macrofactores más importantes se encuentran los cambios climáticos - como el calentamiento global, los fenómenos del Niño/Oscilación del Sur (ENOS) y de la Niña (AENOS) (4)-, que influyen en la intensidad y duración de las temporadas de lluvias y huracanes o provocan intensas sequías y daños a la biodiversidad. Estos cambios causan alteraciones en los ecosistemas y se crean las condiciones ideales que facilitan la expansión y diseminación de organismos patógenos y sus vectores $(5,6)$. Otros macrofactores son el crecimiento poblacional, las migraciones y la urbanización no controlada, que provocan el crecimiento de las ciudades, con cinturones de pobreza y falta de servicios básicos, especialmente de los relacionados con el suministro de agua y la eliminación de residuales líquidos y sólidos $(7,8)$. Estos trastornos traen consigo el aumento en el número de criaderos de vectores, entre ellos del mosquito Aedes aegypti, principal vector del virus del dengue. Por otra parte, los microfactores dependen de las características del virus (9), del vector (mosquito) y su creciente resistencia a los insecticidas $(10,11)$ y del huésped (la persona susceptible) (12). Los microfactores influyen estrechamente en el comportamiento de la enfermedad y la presentación de formas graves del dengue.

La pérdida de la capacidad de respuesta para prevenir y controlar el dengue por parte de los programas nacionales constituye otro factor determinante de la expansión de esta enfermedad. En el presente trabajo se ofrece un análisis de la Estrategia de Gestión Integrada para la Prevención y el Control del Dengue (EGIdengue), aprobada en el $44 .^{\circ}$ Consejo Directivo de la Organización Panamericana de la Salud, y de sus principales resultados preliminares. 


\section{ANTECEDENTES}

Las campañas de erradicación del $A$. aegypti fueron muy exitosas en la década de 1950 y 1960 y para el año 1972 se había logrado erradicar el vector en 21 países de las Américas (13). Sin embargo, en los últimos 22 años, la incidencia de dengue en la Región ha tenido una tendencia ascendente, con picos epidémicos cada vez mayores que se repiten cada 3-5 años casi de manera regular. Esto puede deberse a la acumulación, en ese lapso, de grandes grupos poblacionales susceptibles a la infección, a la circulación de diferentes serotipos del virus, al aumento de la virulencia y la patogenicidad de las cepas circulantes (9) y a la diseminación del vector a nuevas áreas de la Región y del mundo (14).

En la actualidad se informan casos de dengue en más de 30 países de las Américas; entre 2001 y 2005 se notificaron 2879926 casos de dengue, de ellos 65235 de dengue hemorrágico y 789 muertes. El mayor número de casos en este período corresponde a Brasil, Colombia, Venezuela, Costa Rica y Honduras (82\% del total). Sin embargo, el mayor riesgo de enfermar por dengue lo tienen los países del Caribe, ya que poseen las tasas de inciden-cia más elevadas por tener menor población. En 2005, la Guyana Francesa, Martinica, Guadalupe y Surinam presentaron las mayores tasas de incidencia por 100000 habitantes (2567, 1575, 780 y 680, respectivamente) (15).

Hasta hace pocos años, los modelos para el control del dengue no eran suficientemente integrados y participativos y no abordaban el problema en toda su magnitud y dimensiones. Su principal deficiencia era que no rebasaban las fronteras del sector salud, por lo que no tenían alcance intersectorial. La falta de un tratamiento específico y de una vacuna efectiva contra el dengue obliga a diseñar nuevas estrategias que rompan los esquemas verticales y paternalistas tradicionales y que impulsen cambios conductuales y el empoderamiento de los ciudadanos según las particularidades de cada país.

\section{ESTRATEGIA DE GESTIÓN INTEGRADA PARA LA PREVENCIÓN Y EL CONTROL DEL DENGUE}

El dengue ha estado presente en la agenda política de la Organización Mundial de la Salud (OMS) y de la Organización Panamericana de la Salud (OPS) durante décadas, y esta atención se ha incrementado en los últimos años (16-20).
En 1999, la OMS presentó una estrategia mundial para la prevención y el control del dengue y el dengue hemorrágico $(17,18)$. En 2001, la OPS formuló las directrices regionales para la prevención y el control del dengue, contenidas en la Resolución CD43.R4 de su Consejo Directivo (19). Estos lineamientos, que retomaban los publicados por la OPS en 1994 (16), perseguían promover una nueva generación de programas para reforzar los mecanismos de prevención y control del dengue y los programas nacionales mediante la participación comunitaria, la educación sanitaria y la coordinación de acciones extrasectoriales, ya fuera con organizaciones gubernamentales o no gubernamentales.

En el año 2003, durante el $44 .^{\circ}$ Consejo Directivo de la OPS/OMS, se aprobó la Resolución CD44.R9, cuyo objetivo era promover un cambio en el enfoque de los programas nacionales (20). Esa resolución contenía nuevas recomendaciones sobre las formas y los métodos que se debían aplicar a la cooperación técnica de la Organización, introducía el modelo de la EGI-dengue y creaba un grupo técnico de trabajo sobre el dengue en la Región (GTdengue internacional).

\section{EGI-dengue: un nuevo modelo de gestión}

La EGI-dengue es un modelo de gestión que tiene como objetivo fortalecer los programas nacionales con vistas a reducir la morbilidad, la mortalidad y la carga social y económica generada por los brotes y las epidemias de dengue. Para alcanzar sus objetivos, esta estrategia busca modificar la conducta de las personas y de la comunidad de manera que disminuyan los factores de riesgo de transmisión con medidas coordinadas tanto dentro como fuera del sector salud. Además, llama a elaborar y ejecutar planes nacionales y subregionales integrados que permitan trazar una estrategia nacional sostenible, diseñada por las autoridades y los especialistas de cada país con la cooperación técnica del GT-dengue internacional. Estas estrategias nacionales y regionales deben tener un enfoque interprogramático, integrado e intersectorial, basado en una nueva práctica, que permita evaluar y dar continuidad a las acciones con recursos nacionales (21).

El GT-dengue internacional no es más que un grupo de expertos que, partiendo del análisis regional, se incorporan al trabajo del equipo técnico de los países para apoyar la elaboración de las EGIdengue nacionales. De esta forma, la colaboración técnica brindada por el Programa Regional de Dengue de la OPS/OMS —con una visión multidisciplinaria e integradora - tiene el objetivo 
concreto de orientar los esfuerzos estratégicos nacionales para implementar y desarrollar las recomendaciones de la Resolución CD43.R4, bajo los principios de la solidaridad, el respeto, la equidad, la integridad y la excelencia.

La EGI-dengue agrupa áreas de acción y componentes clave que tienen la mayor responsabilidad en la planificación y ejecución de las actividades de prevención y control del dengue. Entre esas áreas clave se encuentran la comunicación social, la vigilancia epidemiológica, el servicio de laboratorio, la atención al paciente, los servicios entomológicos y el cuidado del medio ambiente (figura 1). De acuerdo con las realidades nacionales, los expertos de cada país deciden si se deben tomar en cuenta otros componentes.

El proceso de elaboración de la EGI-dengue en un país es un ejercicio metodológico que se inicia con la abogacía y la coordinación con las autoridades nacionales para establecer grupos de trabajo nacionales y lograr un consenso técnico y político sobre las condiciones necesarias para ese proceso. Primeramente se debe realizar un diagnóstico de las fortalezas, las oportunidades, las debilidades y las amenazas del programa nacional de dengue, según cada uno de sus componentes y tomando como

\section{FIGURA 1. Componentes de la Estrategia de Gestión Integrada para la Prevención y el Control del Dengue en la Región de las Américas}

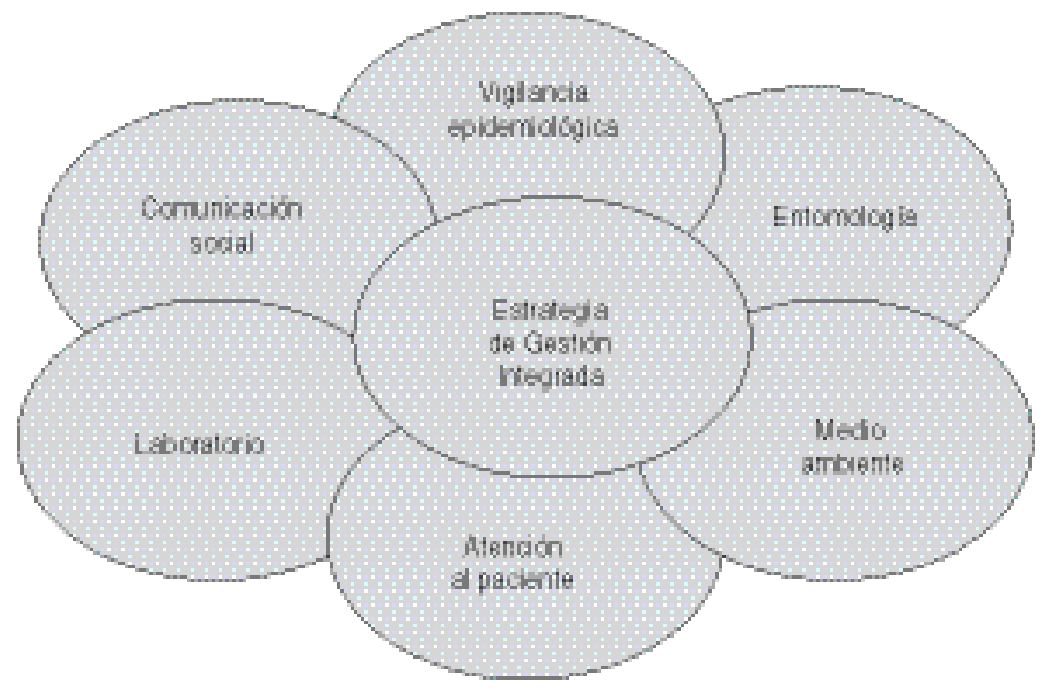

CUADRO 1. Bases de la Estrategia de Gestión Integrada para la Prevención y el Control del Dengue (EGI-dengue), ajustada para América Central y República Dominicana (EGI-CA-DOR)

\begin{tabular}{|c|c|}
\hline Meta & Contribuir a la reducción de la morbilidad, la mortalidad y la carga socioeconómica provocada por el dengue. \\
\hline Propósito & $\begin{array}{l}\text { Elaborar, implementar y consolidar una estrategia de gestión integrada y multisectorial para la prevención y el } \\
\text { control del dengue en los países de América Central y la República Dominicana. }\end{array}$ \\
\hline \multirow[t]{3}{*}{ Indicadores } & $\begin{array}{l}\text { Disminuir en } 50 \% \text { la tasa de incidencia de dengue en un período de } 5 \text { años en los países centroamericanos } \\
\text { que cuentan con una EGl-dengue. }\end{array}$ \\
\hline & $\begin{array}{l}\text { Reducir y mantener la tasa de mortalidad por dengue hemorrágico por debajo de } 2 \% \text { anual en todos los } \\
\text { países. }\end{array}$ \\
\hline & $\begin{array}{l}\text { Incorporar a la estrategia integrada de prevención y control del dengue al menos a } 70 \% \text { de los municipios de } \\
\text { cada país, a los sectores salud, gobierno, educación y a las ONG. }\end{array}$ \\
\hline \multirow[t]{3}{*}{ Fuentes de verificación } & Planes y agenda de la EGI-CA-DOR. \\
\hline & Informes de monitoreo y evaluación a niveles nacional y subregional. \\
\hline & Planes de trabajo operativos integrados de todos los componentes. \\
\hline \multirow[t]{3}{*}{ Condiciones previas } & Voluntad política para implementar y desarrollar la EGI-CA-DOR. \\
\hline & $\begin{array}{l}\text { Disponibilidad de recursos humanos, financieros y logísticos para la implementación de la EGI-CA-DOR en } \\
\text { los países del área. }\end{array}$ \\
\hline & Compromiso institucional, interinstitucional, intersectorial y comunitario en los países. \\
\hline
\end{tabular}


CUADRO 2. Resultados esperados de la Estrategia de Gestión Integrada para la Prevención y el Control del Dengue para América Central y la República Dominicana

\begin{tabular}{|c|c|}
\hline Resultados esperados & Actividades \\
\hline $\begin{array}{l}\text { Saneamiento ambiental: contar con } \\
\text { un grupo de trabajo ambiental } \\
\text { multisectorial integrado y con } \\
\text { acciones que permitan reducir los } \\
\text { factores ambientales de riesgo de } \\
\text { transmisión del dengue }\end{array}$ & $\begin{array}{l}\text { A1. Promover la revisión, actualización y aplicación de las leyes y reglamentos que rigen: } \\
\text { - el abastecimiento de agua } \\
\text { - el manejo adecuado de desechos sólidos } \\
\text { - la comercialización de neumáticos usados } \\
\text { - la disposición final de neumáticos desechados } \\
\text { - los planes de urbanización. }\end{array}$ \\
\hline $\begin{array}{l}\text { Epidemiología: fortalecer los } \\
\text { sistemas nacionales de vigilancia } \\
\text { integral del dengue (epidemio- } \\
\text { lógica, entomológica, de labora- } \\
\text { torio y de atención al paciente, } \\
\text { con promoción y saneamiento } \\
\text { ambiental) y su funcionamiento } \\
\text { eficaz, eficiente y oportuno }\end{array}$ & $\begin{array}{l}\text { A1. Estandarizar las definiciones de caso y ajustar, imprimir y divulgar los protocolos. } \\
\text { A2. Definir los indicadores mínimos de cada uno de los componentes (epidemiológicos, } \\
\text { entomológicos, de laboratorio, de atención al paciente, de promoción y de saneamiento } \\
\text { ambiental) que alimenten el sistema de vigilancia integral. } \\
\text { A3. Notificar oportunamente los casos, según el flujo de información establecido en cada país. } \\
\text { A4. Utilizar los espacios subregionales establecidos de comunicación (páginas en Internet, } \\
\text { plataforma de INFOCOM y el LISTSERVER de la Red Centroamericana de Enfermedades } \\
\text { Emergentes y Reemergentes). }\end{array}$ \\
\hline $\begin{array}{l}\text { Laboratorio: fortalecer la red de } \\
\text { laboratorios integrados al sistema } \\
\text { de vigilancia epidemiológica de } \\
\text { dengue, con capacidad de generar } \\
\text { información oportuna y de calidad }\end{array}$ & $\begin{array}{l}\text { A1. Integrar el laboratorio como elemento de base al sistema de vigilancia de dengue. } \\
\text { A2. Establecer los criterios epidemiológicos y de laboratorio para la toma de muestras } \\
\text { biológicas, según la situación epidemiológica. } \\
\text { A3. Fortalecer la capacidad de respuesta de los laboratorios clínicos y de diagnóstico de } \\
\text { dengue en los países. }\end{array}$ \\
\hline $\begin{array}{l}\text { Entomología: controlar y eliminar } \\
\text { los criaderos de } A \text {. aegypti }\end{array}$ & $\begin{array}{l}\text { A1. Aplicar medidas antivectoriales para el control de los criaderos mediante métodos físicos, } \\
\text { biológicos y químicos y desarrollar técnicas innovadoras. } \\
\text { A2. Aplicar sustancias adulticidas en las localidades donde haya un incremento de personas } \\
\text { con fiebre o del índice de mosquitos adultos o pupas, así como en zonas de transmisión } \\
\text { endémica y en situaciones epidémicas. } \\
\text { A3. Establecer unidades entomológicas operativas a nivel central, regional y local. }\end{array}$ \\
\hline $\begin{array}{l}\text { Promoción de la salud y comuni- } \\
\text { cación social: elaborar, ejecutar } \\
\text { y evaluar planes de comunicación } \\
\text { para lograr cambios de conducta } \\
\text { (COMBI) en los países cubiertos } \\
\text { por la EGI-CA-DOR }\end{array}$ & $\begin{array}{l}\text { A1. Promover alianzas estratégicas con el sector privado, organizaciones sociales, medios de } \\
\text { comunicación y comunidades e instituciones públicas para ejecutar acciones conjuntas de } \\
\text { promoción, prevención y control. } \\
\text { A2. Elaborar, ejecutar y evaluar planes COMBI y planes de participación comunitaria y de } \\
\text { movilización social. } \\
\text { A3. Intercambiar experiencias e información entre los países cubiertos por la EGI-CA-DOR. } \\
\text { A4. Elaborar, evaluar y difundir lineamientos técnicos e instrumentos que permitan } \\
\text { estandarizar los procesos de participación comunitaria y de movilización social en dengue. }\end{array}$ \\
\hline $\begin{array}{l}\text { Atención al paciente: diagnosticar y } \\
\text { tratar oportunamente a los } \\
\text { enfermos de dengue }\end{array}$ & $\begin{array}{l}\text { A1. Revisar, actualizar y difundir protocolos nacionales de diagnóstico y tratamiento de los } \\
\text { pacientes con dengue y dengue hemorrágico para todos los niveles de atención. } \\
\text { A2. Implementar y ejecutar los planes de contingencia para el abordaje clínico en situaciones } \\
\text { de brote. }\end{array}$ \\
\hline $\begin{array}{l}\text { Investigación y capacitación: } \\
\text { desarrollar investigaciones } \\
\text { técnicas, operativas y formativas y } \\
\text { capacitar los recursos humanos } \\
\text { disponibles }\end{array}$ & $\begin{array}{l}\text { A1. Definir y ejecutar las líneas de investigación básica en función de las necesidades de cada } \\
\text { componente. } \\
\text { A2. Formar equipos multidisciplinarios para planificar y ejecutar las líneas de investigación } \\
\text { seleccionadas. } \\
\text { A3. Desarrollar talleres de capacitación para el personal participante en los diferentes } \\
\text { componentes de la EGI-dengue. }\end{array}$ \\
\hline
\end{tabular}

referencia las experiencias históricas y las lecciones aprendidas en el control de la enfermedad en el país.

Luego de la incorporación del GT-dengue internacional al país, se realiza un taller metodológico de expertos nacionales e internacionales y se establece un marco lógico (matriz) de la EGI-dengue, como el estructurado para América Central y la República Dominicana (cuadros 1 y 2). El hecho de que el documento esté elaborado en forma de matriz permite al equipo de trabajo integrar funcionalmente actividades y tareas específicas entre los diferentes componentes. Este documento de trabajo - que contiene los elementos de monitoreo y evaluación- permite, además, 
añadir y modificar acciones para alcanzar los objetivos propuestos. Otros valores agregados de la construcción matricial del documento de la EGIdengue es que permite hacer comparaciones con procesos similares desarrollados en otros países y con las estrategias subregionales. El hecho de incorporar cálculos financieros hace más fácil la negociación con los donantes externos.

Una vez logrado un sólido documento técnico de la EGI-dengue, se presenta y analiza con las máximas autoridades del sector salud y se elabora una propuesta de agenda de implementación a la cual se le debe dar seguimiento.

\section{Implementación de la EGI-dengue en las Américas}

Hasta el momento, 11 países de la Región han elaborado o implementado sus estrategias nacionales de gestión integrada para la prevención y control del dengue: Brasil, Colombia, Costa Rica, El Salvador, Guatemala, Honduras, Nicaragua, Panamá, Paraguay, República Dominicana y Venezuela (cuadro 1). Además, se elaboró una estrategia subregional para América Central y República Dominicana (EGI-CADOR $)^{2}$, con la participación de expertos de todos los países del área y consultores de la OPS/OMS. La EGICA-DOR ha servido de guía para elaborar las estrategias nacionales y ha permitido homogenizar las acciones técnicas, de acuerdo con las particularidades de esta área geográfica.

Entre los componentes que contribuyen a que las EGI-dengue alcancen mejores resultados en los países se encuentran los planes conocidos como Comunicación para Lograr Cambios de Conducta (COMBI) aplicados a la lucha contra el dengue y su vector. Estos planes se basan en una metodología diseñada para ayudar de manera estructurada a planificar, poner en práctica y monitorear acciones de movilización y comunicación social dirigidas a lograr y mantener cambios conductuales específicos que eleven el grado de prevención y control de las enfermedades transmisibles, en particular del dengue. Hasta el momento se han elaborado 22 planes COMBI en la Región ${ }^{3}$, relacionados con la manipulación adecuada y la limpieza de los contenedores domésticos de agua, la disposición de los neumáticos desechados, el manejo de los desechos sólidos y el aumento de la capacidad de reconocimiento de la enfermedad, tanto por el personal médico como por el mismo paciente (22-30).

\footnotetext{
2 Véanse más detalles en http://www.paho.org/common/Display. asp?Lang=S\&RecID $=6502$

3 Véanse más detalles en http://www.paho.org/Spanish/AD/DPC/ $\mathrm{CD} /$ den-bid-idb-pjt.htm
}

\section{RESULTADOS PRELIMINARES OBTENIDOS}

En todos los países en los que se ha implementado la EGI-dengue se ha producido un intercambio constante entre las áreas técnicas y los responsables de tomar las decisiones en los ministerios y los municipios. Esto ha permitido generalizar y difundir las experiencias y lecciones aprendidas en cada uno de los diferentes componentes de la EGI-dengue y ha llevado al fortalecimiento progresivo de estas áreas. También se observa una mayor apertura del personal del sector salud - tanto profesional como técnico-, lo que ha permitido controlar otros componentes e integrarlos al proceso de planificación, algo que va mucho más allá de la mera intervención de control vectorial que tradicionalmente se ha venido haciendo.

Las EGI nacionales plasman las actividades más importantes y contribuyen a abordar el problema del dengue desde una perspectiva de salud más amplia y a que las actividades se ejecuten según el documento marco. Esto evita la improvisación y hace que las acciones se basen en un sólido enfoque técnico, muy necesario para el control del dengue. La planificación de estas actividades se realiza mediante discusiones de los grupos de trabajo de dengue, tanto nacionales como internacionales, y constituye un esfuerzo importante y sin precedente de integración dentro de cada país, aunque todavía no se ha logrado integrar plenamente todos los componentes.

En los países que se encuentran en el proceso de implementación de la EGI-dengue se han constatado algunos resultados positivos, cada vez con mayor fuerza, entre ellos:

- una mayor coordinación dentro de los ministerios, tanto desde el punto de vista técnico como gerencial;

- una mayor coordinación con otros sectores, especialmente con los municipios;

- una mejor coordinación con grupos comunitarios organizados y la puesta en práctica de nuevos proyectos locales de comunicación para cambiar los hábitos y conductas relacionados con el dengue, como la metodología COMBI;

- un aumento en la capacidad de movilización de recursos y un mayor ajuste del trabajo al marco lógico convenido, lo que obliga a tomar en cuenta los costos de las actividades y tareas y facilita la negociación con posibles donantes;

- el desarrollo de nuevas destrezas y habilidades en temas de participación comunitaria, educación e investigación antropológica;

- un aumento en la capacidad de respuesta y en la incorporación de nuevas herramientas para la vigilancia epidemiológica, como el levantamiento de índices aédicos rápidos (LIRA) en Brasil y las nuevas trampas Mosquitrap para calcular el índice de $A$. aegypti adultos. 
CUADRO 3. Avances de la Estrategia de Gestión Integrada para la Prevención y el Control del Dengue en las Américas

\begin{tabular}{|c|c|c|c|}
\hline Fase actual & País/grupo de países & $\begin{array}{l}\text { Fecha de elaboración } \\
\text { o implementación }\end{array}$ & Financiamiento externo \\
\hline \multirow[t]{11}{*}{ Implementación } & $\begin{array}{l}\text { EGI subregional para } \\
\text { América Central y la } \\
\text { República Dominicana }\end{array}$ & Enero de $2004^{a}$ & OPS/OMS, BID \\
\hline & Nicaragua & Marzo de 2004 & OPS/OMS, BID \\
\hline & Guatemala & Agosto de 2004 & OPS/OMS, BID \\
\hline & Honduras & Septiembre de 2004 & OPS/OMS, BID \\
\hline & República Dominicana & Noviembre de 2004 & OPS/OMS, BID \\
\hline & Costa Rica & Diciembre de 2004 & OPS/OMS, BID \\
\hline & El Salvador & Febrero de 2005 & OPS/OMS, BID \\
\hline & Panamá & Julio de 2005 & OPS/OMS, BID \\
\hline & Venezuela & Mayo de 2004 & OPS/OMS, CIDA ${ }^{c}$ \\
\hline & Paraguay & Mayo de 2005 & OPS/OMS, CIDA \\
\hline & Colombia & Marzo de 2006 & OPS/OMS, CIDA \\
\hline \multirow[t]{3}{*}{ Elaboración } & Perú & Marzo de 2007 & OPS/OMS, CIDA \\
\hline & Argentina & Mayo de 2007 & OPS/OMS y fondos nacionales \\
\hline & $\begin{array}{l}\text { EGI subregional para los } \\
\text { países miembros y } \\
\text { partes de MERCOSUR }\end{array}$ & Abril de 2007 & OPS/OMS \\
\hline
\end{tabular}

Todos los países que han elaborado sus EGIdengue nacionales se encuentran en el proceso de su implementación (cuadro 3). A continuación se presentan algunos de los resultados específicos más significativos por países.

\section{Brasil}

En Brasil se ha reemplazado el tradicional Programa de Erradicación del Aedes aegypti (PEA), por una EGI-dengue nacional que se ha extendido a la totalidad de sus municipios. Desde el año 2004 se organiza el Curso Internacional de Gestión Integrada, dirigido a los niveles gerenciales, que comenzó por los municipios priorizados. Mediante estos cursos, expertos nacionales recibieron capacitación en todos los componentes de la EGI-dengue, incluida la metodología COMBI. Se han desarrollado cuatro planes piloto COMBI y se analiza la posibilidad de extender y generalizar esta metodología. Como ejemplo de trabajo extrasectorial, se realizó una excelente coordinación entre la industria privada (la Asociación Nacional de la Industria del Neumático y su sindicato), la Secretaría de Calidad del Ambiente en los Asentamientos Humanos del Ministerio de Medio Ambiente y la propia comunidad, que culminó con la creación de una red de centros de recepción de neumáticos inservibles (ya que son frecuentes criaderos del vector) denominados Ecopuntos y plantas de resección y corte, ya sea para usarlos como combustible, laminarlos para fabricar zapatos o para convertirlos en asfalto. Hasta el momento se han reciclado más de 70 millones de neumáticos.

\section{Colombia}

La EGI-dengue ha propiciado una mejor articulación entre la Dirección de Salud Pública del Ministerio de la Protección Social y el Instituto Nacional de Salud en Colombia. Además, ha servido como modelo de planeación para otras enfermedades, como la leishmaniasis visceral y la malaria, con un solo modelo integrador en el país. El proceso se ha extendido a las regiones Costa Pacífica, Costa Atlántica, Centro Oriental, el Departamento Archipiélago de San Andrés y Providencia, y el Municipio de Medellín. Gracias a la EGI-dengue se ha logrado una buena coordinación entre la Dirección de Salud Pública y el Ministerio del Ambiente, Vivienda y Desarrollo Sostenible, y en varios departamentos de la Costa Pacífica y en Medellín se ha facilitado la concertación de compromisos de trabajo con otros sectores clave para el desarrollo, como los de educación, servicios y obras públicas. En Colombia ya se cuenta con un plan COMBI nacional (22). 


\section{Costa Rica}

La EGI-dengue se ha extendido a todo el país en los tres niveles de gestión mediante un proceso participativo, que han liderado el Ministerio de Salud y la Caja Costarricense del Seguro Social, y al que se han incorporado los gobiernos locales y la comunidad. Se desarrolló un plan COMBI (23) en Punta Arenas y las evaluaciones realizadas reflejan cambios conductuales positivos en la población. En la región Pacífico Central mejoró notablemente la capacidad de respuesta ante los brotes de dengue y se estableció un sistema de vigilancia epidemiológica. La EGI-dengue recibió apoyo financiero del Gobierno de Japón.

\section{El Salvador}

Actualmente, a la EGI-dengue se han incorporado técnicos y tomadores de decisión del Ministerio de Salud y de algunos municipios en El Salvador. Se fortaleció el diagnóstico de laboratorio, clínico y entomológico a fin de lograr un análisis epidemiológico integral y orientar las acciones de campo. Se ha fortalecido el trabajo con las alcaldías municipales y durante el último año se han puesto en práctica ordenanzas municipales para la lucha contra el dengue. Una experiencia exitosa (producto de un plan COMBI) para la disposición de neumáticos desechados en el municipio de San Martín se extenderá a otros municipios del país (24). La Representación de la OPS/OMS en El Salvador está apoyando la integración de los programas de lucha contra la diarrea y la desnutrición a la EGI-dengue, con un enfoque único que abarque múltiples enfermedades.

\section{Guatemala}

En Guatemala, la EGI-dengue se ha extendido a departamentos y municipios, lo que ha facilitado las negociaciones del Ministerio de Salud con los gobiernos municipales y las organizaciones no gubernamentales (ONG) y comunitarias para el desarrollo de un proyecto COMBI (25) y para extender esta estrategia a todo el país. Ha mejorado el control del dengue en los niveles intermedios y las acciones de prevención, vigilancia y alerta epidemiológica se mantienen activas sin esperar que ocurra un brote. Con los programas de la EGIdengue se ha logrado movilizar recursos financieros del Club Rotario, la Unión Europea y el Gobierno de España.

\section{Honduras}

En Honduras se han creado comisiones extrasectoriales multidisciplinarias, como la Comisión de Ambiente, la de Regulación, la de Educación y Promoción y la de Vigilancia. En los departamentos ya se ajusta la estrategia nacional a sus realidades locales y a los recursos disponibles. Además, la EGI-dengue se ha sumado a la estrategia de promoción de municipios saludables con la finalidad de beneficiar a la población con una mejor calidad de vida. En el país se cuenta con un plan COMBI (26).

\section{Nicaragua}

En algunos departamentos de Nicaragua, como Masaya, la implementación de la EGI-dengue ha contribuido a reorientar las actividades de los Sistemas Locales de Atención Integral de Salud (SILAIS) hacia los municipios, haciendo hincapié en el mejoramiento de la salud ambiental, la reorganización de la estructura del mercado municipal y el mejoramiento de la red de distribución de agua. También se mejoró el monitoreo del uso de la cartilla de dengue, utilizada en las escuelas primarias por orientación del Ministerio de Educación. En los barrios donde se implementó el plan COMBI se crearon espacios de participación que antes no existían, con el concurso de las iglesias y los gobiernos municipales (27), y se ha constatado la disminución de los niveles de transmisión de dengue en los últimos dos años.

\section{Paraguay}

En Paraguay se ha fortalecido la integración y la articulación del sector salud con otros sectores, especialmente con las autoridades municipales y las comisiones vecinales, el Ministerio de Ambiente, las entidades de seguridad social y las Fuerzas Armadas. La respuesta al brote de dengue ocurrido en 2006 fue mucho más sólida y consistente que la observada en años anteriores.

En general, en la Región se continúa actualmente con el proceso de elaboración de las estrategias nacionales y subregionales (cuadro 3) y se espera que durante el primer semestre de 2007 queden listas las EGI-dengue nacionales de Perú y Argentina, así como la estrategia subregional para los estados miembros y partes del MERCOSUR.

\section{CONCLUSIONES}

Se espera que la implementación de la EGI-dengue en los países de la Región permita dar un salto cualitativo hacia estrategias nacionales de prevención y control del dengue basadas en asociaciones más fuertes entre el Estado, sus diferentes ministerios y cuerpos de gobierno en todos los niveles, la empresa privada y las diversas asociaciones comunitarias y civiles. Esta estrategia, una 
vez implementada, permitirá reducir los factores de riesgo de transmisión del dengue, establecer un sistema de vigilancia epidemiológica integral, reducir las poblaciones de $A$. aegypti, mejorar la preparación a los laboratorios para detectar e identificar el virus, optimizar el diagnóstico y el tratamiento de los casos y, en consecuencia, disminuir la frecuencia, magnitud y gravedad de los brotes y epidemias de dengue.

El próximo paso debe ser evaluar las estrategias nacionales en proceso de implementación. Para ello se deben comparar los datos epidemiológicos (número de brotes, de casos y de muertes) y entomológicos en los países antes y después de la implementación de la Estrategia. Además, se debe comprobar que la EGI-dengue se ha incorporado plenamente a los programas nacionales de salud pública. Un instrumento de evaluación se ha elaborado y está en proceso de ser evaluado por el GTdengue antes de su implementación.

Entre los retos pendientes se encuentran consolidar las estrategias ya establecidas, obtener fondos para su total implementación y elaborar y poner en práctica nuevas estrategias en los países que aún no cuentan con ella, así como desarrollar investigaciones operativas para verificar los procesos en marcha. La OPS/OMS continuará llevando a cabo un proceso de promoción a fin de lograr una mayor voluntad política y los fondos necesarios para apoyar y dar mayor sostenibilidad a las estrategias de control y prevención del dengue en nuestra Región.

Agradecimientos. La elaboración de las estrategias nacionales y la subregional para América Central ha contado con el financiamiento conjunto de la OPS/OMS y el Banco Interamericano de Desarrollo (BID). En América del Sur se contó con el financiamiento conjunto de la OPS/OMS y la Agencia Canadiense para el Desarrollo Internacional (CIDA). Se agradece la disposición de las autoridades de salud y la dedicación de los grupos nacionales e internacional para hacer posible estos avances.

\section{SYNOPSIS}

\section{Integrated Strategy for Dengue Prevention and Control in the Region of the Americas}

During the last 22 years, the Region of the Americas has seen an upward trend in dengue incidence, with epidemics peaking ever higher and recurring every 3-5 years, almost regularly. A major factor in the spread of the disease has been the diminished capacity of national programs to respond with dengue prevention and control. This paper evaluates the Integrated Strategy for Dengue Prevention and Control-approved by the 44th Directing Council of the Pan American Health Organization in September 2003-and its preliminary results. The Integrated Strategy for Dengue is a management model designed to strengthen national programs, with a focus on reducing morbidity, mortality, and the societal and economic burdens produced by outbreaks and epidemics. Currently, 11 of the countries in the Region have developed a plan for or implemented a national strategy. In addition, a subRegional plan has been developed for Central America and the Dominican Republic. The Integrated Strategy for Dengue is expected to produce a qualitative leap forward in prevention and control through stronger partnerships among the State, its various ministries, and governing bodies, at all levels; private companies; and the range of community and civil groups. Once implemented, this strategy will reduce risk factors for dengue transmission, establish an integrated epidemiological surveillance system, decrease Aedes aegypti mosquito populations, prepare laboratories to better detect and identify the virus, optimize diagnosis and treatment, and, as a result, decrease the frequency, magnitude, and severity of dengue outbreaks and epidemics.

Key words: dengue, regional health planning, policy making, primary prevention, Americas.

\section{REFERENCIAS}

1. Gubler DJ. Epidemic dengue/dengue hemorrhagic fever as a public health, social and economic problem in the 21st century. Trends Microbiol. 2002(10): 100-3.

2. Cazelles B, Chávez M, McMichael AJ, Hales S. Nonstationary influence of El Nino on the synchronous dengue epidemics in Thailand. PLoS Med. 2005; 2(4):e106.
3. Guzmán MG, Kourí G. Dengue: an update. Lancet Infect Dis. 2002;(2): 33-42.

4. Anyamba A, Chretien JP, Small J, Tucker CJ, Linthicum KJ. Developing global climate anomalies suggest potential disease risks for 2006-2007. Int J Health Geogr. 2006;28;5(1):60.

5. Patz JA, Epstein PR, Burke TA, Balbus JM. Global climate change and emerging infectious diseases. J Am Med Assoc. 1996;275(3):217-23.

6. Khasnis AA, Nettleman MD. Global warming and infectious disease. Arch Med Res. 2005;36(6):689-96.

7. Tauil PL. Urbanização e ecologia do dengue. Cad Saude Publica. 2001;17 (Suppl):99-102.

8. Tatem AJ, Rogers DJ, Hay SI. Estimating the malaria risk of African mosquito movement by air travel. Malar J. 2006; 14(5):57. 
9. Holmes EC, Burch SS. The causes and consequences of genetic variation in dengue virus. Trends Microbiol. 2000(8) 74-7.

10. Cui F, Raymond M, Qiao CL. Insecticide resistance in vector mosquitoes in China. Pest Manag Sci. 2006;62(11): 1013-22.

11. Sunaiyana S, Pungasem P, Kasin S. Detection of insecticides resistance status in Culex quinquefasciatus and Aedes aegypti to four major groups of insecticides. Trop Biomed. 2006;23(1):97-101.

12. Chaturvedi U, Nagar R, Shrivastava R. Dengue and dengue haemorrhagic fever: implications of host genetics. FEMS Immunol Med Microbiol. 2006 47(2):155-66.

13. Rodríguez Cruz R. Estrategias para el control del dengue y del Aedes aegypti en las Américas. Rev Cubana Med Trop. 2002;54:189-201.

14. Lounibos LP. Invasions by insect vectors of human disease. Annu Rev Entomol. 2002;47:233-66.

15. Pan American Health Organization. Number of reported cases of dengue and dengue hemorrhagic fever (DHF), Region of the Americas (by country and subregion) [sitio en Internet] Hallado en http://www.paho.org/ english / ad/dpc/cd/dengue.htm. Acceso el 12 de diciembre de 2006.

16. Pan American Health Organization. Dengue and dengue hemorrhagic fever in the Americas: guidelines for prevention and control. Washington, D.C.: PAHO; 1994. (Scientific Publication No. 548.)

17. World Health Organization. Strengthening implementation of the global strategy for dengue fever/dengue hemorrhagic fever prevention and control: report of the informal consultation; 18-20 October 1999; WHO Headquarter, Geneva. Geneva: WHO; 2000. Hallado en http:/ / whqlibdoc.who.int/hq/2000/
WHO_CDS_(DEN)_IC_2000.1.pdf. Acceso el 12 de diciembre de 2006

18. World Health Organization. Dengue prevention and control. Report by the Secretariat. 55th World Health Assembly A55/19, Provisional Agenda Item 13.14 4. Geneva: WHO; 2002. Hallado en http:/ / www.who.int/gb/ebwha/pdf files/WHA55/ea5519.pdf. Acceso el 12 de diciembre de 2006.

19. Pan American Health Organization Dengue and dengue hemorrhagic fever. Resolution CD43.R4. 53th Session of the Regional Committee, 43th Directing Council. Washington, D.C.: PAHO; 2001. Hallado en http://www.paho. org/english/hcp/hct/vbd/newgeneration-resolutions.pdf. Acceso el 12 de diciembre de 2006.

20. Pan American Health Organization. Dengue. Resolution CD44.R9. 55th Session of the Regional Committee, 44th Directing Council. Washington, D.C.: PAHO; 2003. Hallado en http://www paho.org/english/gov/cd/cd44-r9e.pdf. Acceso el 12 de diciembre de 2006.

21. Organización Panamericana de la Salud, Grupo de Trabajo sobre Dengue. Estrategia de Gestión Integrada para la Prevención y el Control del Dengue en la Región de las Américas. 2. a versión. Santa Cruz de la Sierra, Bolivia: OPS; 2003. (OPS/HDM/CD/440.07.)

22. Padilla JL, Ahumada ML, Lozano G, Barrero N, Rey JJ, Escandón S, et al. Plan de movilización y comunicación social para la prevención y control del dengue, Colombia, 2004-2005. Barranquilla, Colombia: OPS; 2004. (OPS/HDM/CD/ 439.07.)

23. Barquero Chávez F, Elizondo ME, Solano Chinchilla T, Sang SL. Plan para impactar la conducta en la prevención y el control del dengue. Propuesta para las regiones Pacífico Central, Chorotega y Huetar Atlántica, Costa Rica. Managua: OPS; 2003. (OPS/HDM/CD / 438.07)
24. República del Salvador, Ministerio de Salud Pública y Asistencia Social. Plan de comunicación social para cambios de comportamiento en la practica de La Untadita, Comunidades del Distrito Italia I y II, Municipio de Tonacatepeque, San Salvador, El Salvador, 2004. Tegucigalpa: OPS; 2003. (OPS/HDM/ CD/433.07.)

25. República de Guatemala, Ministerio de Salud Pública y Asistencia Social. Programa Nacional de Dengue. Plan para el cambio conductual en la aplicación de la correcta técnica de cepillado de pilas y toneles en las 54 localidades de mayor riesgo del Departamento de Zacapa, Guatemala, C.A. Guatemala: OPS; 2003. (OPS/HDM/CD/434.07.)

26. República de Honduras, Secretaría de Salud. Programa Dengue. Plan nacional para impactar la conducta del lavado de pilas y barriles para la prevención y control del dengue en zonas urbanas marginales de Honduras, 2004-2005. Tegucigalpa: $\quad$ OPS; 2003 (OPS/HDM/CD/435.07.)

27. Campos LA, Chamorro V, Lugo E, Acevedo B, Uriza A. Estrategia de comunicación social para cambios de comportamientos sobre dengue. Managua: OPS; 2003 (OPS/HDM/CD/432.07.)

28. Arjona R, Chung A, Griffith M, Cáceres Carrera L. Plan estratégico para impactar la conducta en la prevención y control del dengue. Panamá: OPS; 2003. (OPS/HDM/CD/431.07.)

29. Revello D, Benites E, Véliz L, Espinoza R. Programa de comunicación para el impacto conductual COMBI para la prevención y control del dengue en el Ecuador, años 2004-2005. Quito: OPS; 2004. (OPS/HDM/CD/436.07.)

30. Martínez M, Solís AT, Lara LA. Plan estratégico de prevención de dengue basado en la estrategia NEPRAM y COMBI en República Dominicana [proyecto]. Managua: OPS; 2003. (OPS/ $\mathrm{HDM} / \mathrm{CD} / 437.07$. 